\title{
Study of the piezoresistivity of doped nanocrystalline silicon thin films
}

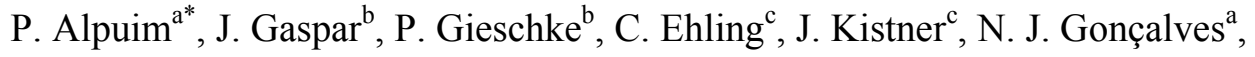 \\ M. I. Vasilevskiy ${ }^{\mathrm{a}}$, and O. Paul ${ }^{\mathrm{b}}$
}

\footnotetext{
${ }^{a}$ Center of Physics, Universidade do Minho, Campus de Azurém, 4800-058 Guimarães, Portugal

${ }^{\mathrm{b}}$ Department of Microsystems Engineering (IMTEK), Microsystems Materials Laboratory, University of Freiburg, Freiburg 79110, Germany

${ }^{c}$ Institut für Physikalische Elektronik, Universität Stuttgart, 70569 Stuttgart, Germany

*corresponding author, e-mail: palpuim@fisica.uminho.pt
}

\begin{abstract}
The piezoresistive response of $n$ - and $p$-type hydrogenated nanocrystalline silicon thin films, deposited by hot-wire (HW) and plasma-enhanced chemical vapor deposition (PECVD) on thermally oxidized silicon wafers, has been studied using four-point bending tests. The piezoresistive gauge factor $(G F)$ was measured on patterned thin-film micro-resistors rotated by an angle $\theta$ with respect to the principal strain axis. Both longitudinal $\left(G F_{L}\right)$ and transverse $\left(G F_{T}\right) G F \mathrm{~s}$, corresponding to $\theta=$ $0^{\circ}$ and $90^{\circ}$, respectively, are negative for $n$-type and positive for $p$-type films. For other values of $\theta\left(30^{\circ}, 45^{\circ}, 120^{\circ}\right.$ and $\left.135^{\circ}\right) G F$ s have the same signal as $G F_{L}$ and $G F_{T}$ and their value is proportional to the normal strain associated with planes rotated by $\theta$ relative to the principal strain axis. It is concluded that the films are isotropic in the growth plane since the $G F$-values follow a Mohr's circle with the principal axes
\end{abstract}


coinciding with those of the strain tensor. The strongest $p$-type pirezoresistive response $\left(G F_{L}=41.0, G F_{T}=2.84\right)$ was found in a film deposited by PECVD at a substrate temperature of $250^{\circ} \mathrm{C}$ and working pressure of 0.250 Torr, with dark conductivity $1.6 \Omega^{-1} \mathrm{~cm}^{-1}$. The strongest $n$-type response $\left(G F_{L}=-28.1, G F_{T}=-5.60\right)$ was found in a film deposited by PECVD at $150^{\circ} \mathrm{C}$ and working pressure of 3 Torr, with dark conductivity $9.7 \Omega^{-1} \mathrm{~cm}^{-1}$.

A model for the piezoresistivity of nc-Si is proposed, based on a mean-field approximation for the conductivity of an ensemble of randomly oriented crystallites and neglecting grain boundary effects. The model is able to reproduce the measured $G F_{L}$ values for both $n$ - and $p$-type films. It fails however to explain the transversal $G F_{T}$ data. Both experimental and theoretical data show that nanocrystalline silicon can have an isotropic piezoresistive effect of the order of $40 \%$ of the maximum response of crystalline silicon.

\section{Introduction}

Hydrogenated nanocrystalline silicon (nc-Si:H) is a thin film semiconductor with increasing technological importance due to its superior properties when compared to its parent material, hydrogenated amorphous silicon (a-Si:H), namely, higher structural stability due to a low hydrogen content, higher electron and hole mobility, possibility to control both band gap and crystalline fraction without a significant change of the nanocrystallite size, and a lower optimal deposition temperature. Due to its low deposition temperature, nc-Si:H can be deposited on new types of substrates such as flexible plastics that can withstand temperatures up to $150^{\circ} \mathrm{C}$ at which the electronic quality of the films can

still be ensured. ${ }^{1-2}$ In turn, flexible substrates pave the way for inexpensive fabrication 
processes, like roll-to-roll deposition. ${ }^{3}$ A number of chemical vapor deposition (CVD) techniques has been studied in order to obtain uniform electronic-device quality nc-Si:H films over large areas at low substrate temperatures, i.e. below $150^{\circ} \mathrm{C}$, and high deposition rates, i.e. above $0.3 \mathrm{~nm} / \mathrm{s}^{4-6}$ The most successful techniques to date are those promoting crystallite nucleation in the gas phase such as standard radio-frequency (13.56 MHz) plasma-enhanced CVD (RF), operated at high pressure (1-10 Torr) and high power (above $\left.150 \mathrm{~mW} / \mathrm{cm}^{2}\right){ }^{7}$ and those providing uniform high-density plasmas at low electron temperature (1-2 eV), such as very-high frequency (30-300 MHz) PECVD (VHF), ${ }^{8}$ inductively coupled plasma (ICP) $\mathrm{CVD},{ }^{9}$ surface wave plasma (SWP) and microwave plasma $(2.45 \mathrm{GHz}) \mathrm{CVD} .{ }^{10}$ However, as the wavelength of the excitation field decreases, the difficulties in up-scaling the process to large areas, as required by the industry, make some of these techniques impractical. A plasma-free, well established technique is hot-wire CVD (HW) where gas molecules pyrolytically decompose at the heated filament surface, which is at $1200-2500^{\circ} \mathrm{C}$. The technique is very efficient in providing atomic hydrogen to the film growing surface, thus promoting crystalline growth of the Si films. ${ }^{11}$ For this reason, nc-Si:H films can be obtained by $\mathrm{HW}$ at comparatively low hydrogen $\left(\mathrm{H}_{2}\right)$ to silane $\left(\mathrm{SiH}_{4}\right)$ gas phase concentration ratio, which allows for an increased deposition rate, since the latter scales approximately linearly with the silane concentration. ${ }^{1}$ The absence of ionic bombardment of the growing film is another advantage for the electronic quality of the films. However, plastic substrates may not withstand the high filament temperatures, particularly when long deposition times are required unless they are placed far away from the hot filament, which in turn lowers the deposition rate. ${ }^{12}$

Similarly to doped crystalline $\mathrm{Si}$, doped nc-Si:H exhibits piezoresistive properties - the change of conductivity upon the application of stress - that can be used in the fabrication of strain gauges and other electromechanical transducers. ${ }^{13,14}$ While the piezoresistive 
effect has been widely studied in crystalline silicon (c-Si) since its discovery in $1954,{ }^{15}$ the literature is rather sparse in what concerns the piezoresistance of nc-Si:H. ${ }^{16-19}$

This is a promising route to explore with potential to make way for entirely new applications based on composites with the mechanical properties of the plastic substrate and the electronic properties of the silicon thin film. The concept of sensitive $\operatorname{skin}^{20}$ for next-generation robotics, when robots will no longer need to operate in structured backgrounds but will adapt their movements to muddled, changing environments, can be one of those applications.

Mechanical stress sensors using the piezoresistive effect have been a central component of many microelectromechanical systems (MEMS) such as pressure sensors, accelerometers, and gyroscopes. ${ }^{21,22}$ In order to achieve device compactness and increased performance, one successful approach to date is to post-process the micromechanical parts on top of the signal conditioning circuitry. Being fully compatible with complementary metal-oxide semiconductor (CMOS) and other integrated circuit (IC) technologies due to its low deposition temperature, as previously demonstrated, ${ }^{23-25}$ because of its piezoresistive effect nc-Si:H is also an important thin-film material to interface with CMOS backend technology.

The piezoresistive response can be defined as the ratio between the relative resistance change, $\Delta R / R_{0}$, to the characteristic strain, $\varepsilon$, that causes that change. Since up to a certain strain level the effect is linear, a characteristic gauge factor can be defined in that region as $G F=\left(\Delta R / R_{0}\right) / \varepsilon$. Under the loading conditions schematically shown in Fig.1, the piezoresistive effect along the $x$ direction (i.e. the longitudinal one) can be described by ${ }^{8}$

$$
\frac{\Delta R}{R_{0}}=\left(\varepsilon_{1}-\varepsilon_{2}-\varepsilon_{3}\right)+\pi_{11} P_{1}+\pi_{12}\left(P_{2}+P_{3}\right)
$$


where $\varepsilon_{i}$ and $P_{i}(i=1,2,3)$ are the principal strain and stress components, respectively ( $\varepsilon_{1} \equiv \varepsilon_{x x}$, etc.) and $\pi_{\mathrm{ij}}$ are the piezoresistive coefficients. The first term in Eq. (1) is a geometrical factor while the remaining terms represent the stress-induced change in conductivity, which will be considered in detail in Section 4. For doped c-Si under similar loading configuration and stress levels comparable to those used in this study, the geometrical term can usually be neglected.

Due to the differences in silicon band structure near the top of the valence band and the bottom of the conduction band, $n$ - and $p$-type c-Si layers show distinct piezoresistive behaviors, as described by the corresponding sets of piezoresistive coefficients. Under applied stress, the deformation-induced modifications in the conduction and valence bands $^{26,27}$ result in specific changes in carrier mobility and/or concentration. Typical values of the piezoresistive coefficients for $n$-type and for $p$-type c-Si are listed in Table I. Assuming that the crystallites in nc-Si:H films are randomly oriented, one can expect the piezoresistive effect to be isotropic. It should also be weaker than in c-Si since only some of the crystallites, with more "favorable" orientation with respect to the principal axes of the applied stress, will contribute strongly to the overall effect. We will show in Section 4 that this is indeed the case. It is known as well that grain boundaries effectively attenuate the $G F$ in multicrystalline materials ${ }^{26}$ but these effects seem to be sample-dependent and are difficult to model.

In this paper, we report on an experimental and theoretical study of the piezoresistive response of $n$ - and $p$-type nc-Si:H films deposited by different CVD techniques on thermally oxidized silicon wafers. The piezoresistive gauge factor was measured on patterned thin-film microresistors rotated by angles $\theta=0^{\circ}, 30^{\circ}, 45^{\circ}, 90^{\circ}, 120^{\circ}$ and $135^{\circ}$ relative to the principal strain axis [see Fig.1-a)]. Assuming that the film material is 
isotropic, the principal axes of the strain and conductivity tensors should coincide. In accordance with this assumption, we found that the piezoresistive response for different resistor orientations has longitudinal and transverse components and their dependence on $\theta$ follows the Mohr circle. We present a model for the piezoresistance in nanocrystalline silicon, which is microscopic for $n$-Si and phenomenological for $p$-Si, making use of a mean-field approximation for the evaluation of the strain effect on the average conductivity of an ensemble of randomly oriented crystalline grains, which semi-quantitatively describes our experimental data.

\section{Experimental procedures}

Doped $n$ - and $p$-type nc-Si:H thin films were deposited on 2 " and 4 " thermally oxidized double sided polished (100) silicon wafers with thickness values in the $300-380 \mu \mathrm{m}$ range. Depositions were carried out by HW-CVD, RF-PECVD (13.56 MHz) and VHF-PECVD (70 MHz). The substrate temperature, $T_{\text {sub }}$, was varied between 150 and $280{ }^{\circ} \mathrm{C}$. Gas phase doping was achieved by adding phosphine $\left(\mathrm{PH}_{3}\right)$ and diborane $\left(\mathrm{B}_{2} \mathrm{H}_{6}\right)$ or trimethylboron $\left(\mathrm{B}\left(\mathrm{CH}_{3}\right)_{3}\right)$ for $n$ - and $p$-doping, respectively, to the reactive gas mixture of $\mathrm{SiH}_{4}$ and $\mathrm{H}_{2}$. A further deposition parameter varied was the hydrogen dilution of silane, HD, defined by $\mathrm{HD}=F_{\mathrm{H} 2} /\left(F_{\mathrm{SiH} 4}+F_{\mathrm{H} 2}+F_{\text {dopant }}\right) \times 100 \%$, where the $F$ 's are gas flow rates. The deposition pressure and electrical power used to dissociate the gases varied with CVD technique and gas-phase doping type.

Samples were named according to the film deposition technique and doping type, plus a serial number. For example $n$-HW\#07 refers to sample \#7, which was deposited by HWCVD and $n$-doped. According to their structure, which, for each CVD technique depends

on the HD used during deposition, ${ }^{1,2,4}$ films could be divided into three groups: amorphous, nanocrystalline and mixed-phase. Only one film was amorphous ( $p$-RF\#02). Two films 
were mixed-phase ( $p$-VHF\#06 and $p$-HW\#08), i.e. their crystalline fraction, $X_{\mathrm{c}}$, measured by Raman spectroscopy, ${ }^{1}$ was less than $70 \%$. All the other films studied were nanocrystalline, i.e. with $X_{\mathrm{c}}>70 \%$. The room-temperature dark conductivity, $\sigma_{\mathrm{d}}$, is mainly determined by the film structure. The nc-Si:H films have higher $\sigma_{\mathrm{d}}$, in the range of $2.8-$ $25.4 \Omega^{-1} \mathrm{~cm}^{-1}$, with $n$-type films showing higher values of $\sigma_{\mathrm{d}}$ for the same doping level and crystallinity, than the $p$-type ones. Mixed phase films have $\sigma_{\mathrm{d}} \approx 0.2-0.6 \Omega^{-1} \mathrm{~cm}^{-1}$ and the aSi:H film has $\sigma_{\mathrm{d}}=4.8 \times 10^{-5} \Omega^{-1} \mathrm{~cm}^{-1}$. The film thicknesses are in the range $150-300 \mathrm{~nm}$, as estimated from the growth rate and deposition time. The growth rate was calculated from thickness measurements using optical interferometry, and confirmed by profilometry, of selected samples deposited under the same experimental conditions as those used for the fabrication of the piezoresistors. In the range of thickness used, the nanocrystalline growth regimen is stable, i.e. the film crystalline structure does not depend on the thickness. Deposition conditions and film properties are summarized in Table II.

The piezoresistive response of the films was studied on lithographically patterned $450-\mu \mathrm{m}-$ long and $500-\mu \mathrm{m}$-wide resistors. The voltage drop between two central electrodes placed $150 \mu \mathrm{m}$ apart, when current was injected and collected between two outer electrodes, in a four-point in-line geometry, was measured. Rectangular chips were fabricated containing 6 micro-resistors rotated by angles $\theta=0^{\circ}, 30^{\circ}, 45^{\circ}, 90^{\circ}, 120^{\circ}$ and $135^{\circ}$ relative to the longitudinal chip axis. The chips were patterned on films deposited onto the central part of $9 \mathrm{~mm}$ wide and roughly $100 \mathrm{~mm}$ long silicon strips cut along one $<110>$ direction from 4 " c-Si wafers (see Fig.1).

In case of chips fabricated on 2" wafers, the samples were diced into squares and attached to a silicon strip using an epoxy resin adhesive. The properties of the epoxy layer have a significant influence on the stress distribution on the test chip surface. To minimize this influence, an adhesive with a high shear strength of about $30 \mathrm{MPa}$ was chosen and the 
thickness of the adhesive layer was minimized in order to maximize the stress at the test chip surface. Moreover, for calibration purposes, the curvature of the chip surface during bending experiments was measured using a laser-deflection setup and confirmed by finite element simulations.

Mechanical in-plane stress was introduced by lifting the position stage of the four-point bending bridge (4PBB) and pressing the silicon strip against the two outer supports, as shown in Figs.1-c) and 2-a). The electrical interconnection of the test chip to a printed circuit board (PCB) was ensured by bond wires. Apart from the bond wires, the PCB is mechanically decoupled from the test chip, thus transmitting negligible mechanical stress to the chip [Fig.2-a)]. Measurements were carried out using an Agilent 4155C parameter analyzer. For each deposition run, seven samples were characterized in average. Due to the width-to-thickness ratio of the strips of $w / t \approx 25$, beam behavior can be assumed ${ }^{27}$ and therefore the load applied by the 4PBB generates normal, uniaxial stress, $\sigma_{\mathrm{xx}}$, in the film. Each longitudinal fiber of the specimen in the region between the inner loading bars was therefore bent to an arc of circle by a constant bending moment. The chip was placed in the outer tensile surface of the beam, and therefore it is in the locus of maximum strain, given by: $:^{28}$

$$
\varepsilon_{1}=\frac{6 t}{2 L_{o}^{2}+6 L_{o} L_{i}}
$$

where $t, \Delta d, L_{o}$, and $L_{i}$ denote the thickness of the wafer strip, the displacement of the inner bars with respect to the outer elements, the distance between the inner and outer loading bars $\left(L_{o}=18 \mathrm{~mm}\right)$, and half the inner inter-bar distance $\left(L_{i}=10 \mathrm{~mm}\right)$, respectively, as schematically represented in Fig.2-a). In our experiment, both displacement of the loading bars and applied force were measured, since it is easier to detect the point of contact between loading bar and the specimen by monitoring the force 
than by looking at the displacement only. Since the applied force, $F$, is divided in equal parts, $F / 2$, between the two inner bars, the following relation between the measured $F$ and the principal strain $\varepsilon_{1}$ in the film along the $x$-axis, is valid: ${ }^{28}$

$$
\varepsilon_{1}=\frac{3 L_{o} C_{\text {glue }}}{E_{110} w t^{2}} F
$$

where $C_{\text {glue }}=0.2273$ is a correction factor added for glued chips, obtained from finiteelement (FE) simulations along with laser-deflection curvature measurements $\left(C_{\text {glue }}=1\right.$ otherwise), and $E_{110}$ is the Young's modulus of silicon along $<110>\operatorname{direction}\left(E_{110}=168.9\right.$ GPa). The normal stress along the $x$-axis is given by $P_{1}=E_{\text {film }} \varepsilon_{1}$ where $E_{\text {film }}$ is the Young's modulus of the film. Moreover, $P_{2}=0$ and $\varepsilon_{2}=-v \varepsilon_{1}$, where $v=0.27$ is the Poisson's ratio of the film. A FE simulation confirmed the assumptions that were based on the four-point bending beam theory for an isotropic material. ${ }^{29}$

\section{Experimental results}

Figure 3 shows the relative resistance change as a function of applied strain observed in $p$ [Fig.3-a)] and $n$-type [Fig.3-b)] nc-Si:H films deposited by HW ( $p$-HW\#09) and RF (nRF\#04), respectively, at $T_{\text {sub }}=150^{\circ} \mathrm{C}$. Gauge factors are both positive for the $p$-type film $\left(G F_{L}=+32.1, G F_{T}=+7.2\right)$ and both negative $\left(G F_{L}=-28.0, G F_{T}=-5.6\right)$ for the $n$-type film. As can be concluded from Fig. 4, which shows a bar chart of GFs of all the films, such piezoresistive response is representative of the behavior of the films studied, irrespective of the film deposition temperature or deposition technique used: $G F \mathrm{~s}$ are always positive for $p$-type and negative for $n$-type films. For the set of films studied, $G F_{L}$ values deviate from their average between $-18.5 \%$ and $+26.3 \%$ for $p$-type, and between $-22.3 \%$ and $+30.4 \%$ for $n$-type. $G F_{T}$ shows an even smaller variability both in $n$ - and $p$ type films. In $p$-type films dark conductivity values span nearly five orders of magnitude, 
from $4.8 \times 10^{-5} \Omega^{-1} \mathrm{~cm}^{-1}$ in sample $p$-RF\#02 to $2.8 \Omega^{-1} \mathrm{~cm}^{-1}$ in sample $p$-HW\#09 (see table II). It is interesting to note that the amorphous film, $p$-RF\#02, with the lowest $\sigma_{\mathrm{d}}$, has $G F_{L}=$ 24.4 which, though being the lowest in the range, is not too different from the $G F_{L}$ values found in $p$-type films with nanocrystalline structure $\left(G F_{L} \approx 30-40\right.$, see Fig.4). An important consequence of this fact is that sensors with high piezoresistive response can be made out of low-temperature, doped silicon films with a resistance that can be fixed at a value chosen from an interval spanning several orders of magnitude. Depending on the particular application, if very low operating currents are required our results show that it is possible to design high impedance sensors using $\mathrm{p}^{+}$-a-Si:H piezoresistors, without loss of sensitivity, when compared to sensors made of $\mathrm{p}^{+}$-nc-Si:H films.

In $n$-type films it was not possible to examine such an extended range of conductivities since the wafer with the chip made of $n$-a-Si:H broke during clean room processing. Therefore, in this work, the range of $n$-type $G F \mathrm{~s}$ is associated with $\sigma_{\mathrm{d}}$ values in the interval going from 2.87 to $25.4 \Omega^{-1} \mathrm{~cm}^{-1}$, a much smaller range than that studied for $p$-type films. Figure 5-a) shows the relative resistance change as a function of principal strain, $\varepsilon_{1}$, for resistors aligned at angles ranging between $0^{\circ}$ and $90^{\circ}$ with the principal strain axis, in an $n$-type film deposited by $\mathrm{RF}$ at $T_{\text {sub }}=250^{\circ} \mathrm{C}$. It is clear that the principal axes of the piezoresistance tensor are coincident with the principal axis of the strain tensor since the two principal values of gauge factor correspond to angles $\theta=0^{\circ}\left(G F_{1}=G F_{L}\right)$ and $\theta=90^{\circ}$ $\left(G F_{2}=G F_{T}\right)$, where the maximum and minimum piezoresistive response of the films is observed, respectively. This shows that the film is isotropic in the XY-plane which is confirmed in Fig. 5-b), where the $G F$ data (solid circles) as a function of resistor angle are well fitted (solid line) by a cosine function: $G F=G F_{\text {offset }}+G F_{\text {amp }} \cos (2 \theta)$. The cosine-of$2 \theta$ dependence of $G F$ (i.e, $G F$ is periodic in $\theta$, with period $\pi$ ) follows the dependence of normal strain on $2 \theta$, according to a Mohr's circle for plane strain. This in-plane isotropy 
was found in all films studied. However the fitting parameters for $G F$, offset and amplitude $\left(G F_{\text {offset }}=-16.8\right.$ and $G F_{\text {amp }}=-8.4$ for sample $n-\mathrm{RF} \# 01$ in Fig.5), are different for different films studied, following their different $G F_{L}$-values. In general, $G F_{L}$ can be correlated with $\sigma_{\mathrm{d}}$ : higher $\sigma_{\mathrm{d}}\left(25 \Omega^{-1} \mathrm{~cm}^{-1}\right.$ for $\left.n-\mathrm{RF} \# 01\right)$ corresponds to stronger piezoresistive response $\left(G F_{L}=-25.2\right)$. The correlation between conductivity and piezoresistivity is found in general both in $n$ - and $p$-type samples (see Table II). Nevertheless, as mentioned above, while $\sigma_{\mathrm{d}}$ may vary orders of magnitude, for example when going from a $p^{+}$-a-Si:H to a $p^{+}-$ nc-Si:H film, the corresponding $G F$ changes only a few units (1-10).

Figure 6-a) shows the piezoresistive response in the dark of resistors aligned at angles in the range $0^{\circ}<\theta<90^{\circ}$ with the principal axis of strain (X-axis) for a $p$-type nc-Si:H film deposited at $250^{\circ} \mathrm{C}$ by VHF (sample $p$-VHF\#05). Similarly to the $n$-type films, the dependence of $G F$ on $\cos (2 \theta)$ [(Fig. 6-b)] follows the normal strain dependence on $2 \theta$. However, both $G F_{L}(41.0)$ and $G F_{T}(2.84)$ and, consequently the fitting parameters $\left(G F_{\text {offset }}\right.$ $\left.=19.7, G F_{\mathrm{amp}}=19.2\right)$, are now positive. Compared to sample $p$-HW\#09 [Fig. 3-a)] deposited by HW, sample $p$-VHF\#05 has lower $\sigma_{\mathrm{d}}\left(1.63\right.$ against $\left.2.80 \Omega^{-1} \mathrm{~cm}^{-1}\right)$ but higher $G F_{L}$ (41 against 32). This is one of the only two exceptions to the correlation between $G F$ and $\sigma_{\mathrm{d}}$ that were observed. The other exception was sample $n$-RF\#01, consisting of an $n$ type film with the highest $\sigma_{\mathrm{d}}$ but not the strongest piezoresistive response.

\section{Model for the piezoresistance of nc-Si}

\subsection{Definitions}

Let us consider a conducting medium that, at rest, can be described by an isotropic conductivity tensor, $\sigma_{i j}=\sigma \delta_{i j}$. Under applied strain, $\varepsilon_{i j}$, the conductivity becomes 
anisotropic and we denote by $\Delta \hat{\sigma}=\Delta \sigma_{i j}$ the variation of the conductivity resulting from the deformation of the medium. Following Ref. 30, we define a rank 4 tensor, $\hat{M} \equiv M_{i j k l}$, according to

$$
\frac{\Delta \sigma_{i j}}{\sigma}=\sum_{k, l} M_{i j k l} \varepsilon_{k l} .
$$

This tensor is symmetric with respect to permutations $(i j) \leftrightarrow(k l), i \leftrightarrow j$ and $k \leftrightarrow l$. Its components can be conventionally denoted as:

$$
M_{x x x x}=M_{y y y y}=M_{z z z z} \equiv M_{11} ; \quad M_{x x y y}=M_{x x z z}=M_{y y z z} \equiv M_{12} ; \quad M_{x y x y}=M_{x z x z}=M_{y z y z} \equiv M_{44} .
$$

These are simply related to the earlier introduced piezoresistance coefficients:

$$
\begin{gathered}
M_{11}+2 M_{12}=-\left(C_{11}+2 C_{12}\right)\left(\pi_{11}+2 \pi_{12}\right) \\
M_{11}-M_{12}=-\left(C_{11}-C_{12}\right)\left(\pi_{11}-\pi_{12}\right) ; \quad M_{44}=-C_{44} \pi_{11}
\end{gathered}
$$

where $C_{a b}$ are elastic constants. Our purpose is to calculate the $M_{a b}$ components since the gauge factors can then be readily evaluated (neglecting the geometrical term) as

$$
G F_{L}=\frac{1}{\varepsilon_{1}} \frac{\Delta R_{L}}{R_{L}}=-\frac{1}{\varepsilon_{1}} \frac{\Delta \sigma_{x x}}{\sigma} ; \quad G F_{T}=\frac{1}{\varepsilon_{1}} \frac{\Delta R_{T}}{R_{T}}=-\frac{1}{\varepsilon_{1}} \frac{\Delta \sigma_{y y}}{\sigma} .
$$

Since the conductivity tensor is diagonal in the same reference frame as the strain tensor, a consequence of the isotropy of the films in the $\mathrm{XY}$ plane, it is possible to construct the Mohr circle in Fig. 7 for the gauge factor using its principal values, $G F_{L}$ and $G F_{T}$, to find the center, $c$, and the radius, $r$, of the circle drawn in the XY plane, in a similar way to that used to draw a Mohr circle for strain: ${ }^{29}$

$$
r=\frac{\left|G F_{L}-G F_{T}\right|}{2} ; \quad c=\frac{G F_{L}+G F_{T}}{2}
$$

By using the Mohr circle for $G F$, it is possible to estimate the piezoresistive response of thin film resistors oriented at any angle relative to the principal strain axis in the usual way: 


$$
\begin{gathered}
G F_{L}^{\prime}=c+\frac{G F_{L}-G F_{T}}{2} \cos 2 \theta \\
G F_{H}^{\prime}=-\frac{G F_{L}-G F_{T}}{2} \sin 2 \theta,
\end{gathered}
$$

where the primes refer to a referential with axis $X^{\prime}$ parallel to the direction of current flow in the resistor rotated by angle $\theta$ and $G F_{H}^{\prime}$ refers to $G F$ measured in a pseudo-Hall geometry, i.e. one where the voltage drop is measured in a direction perpendicular to the current flow in the resistor. The Mohr circle for $G F$ follows directly from the correspondent circle for plane strain and the isotropy of the piezoresistive response of the films in planes perpendicular to the growth direction. Such circles are plotted in Fig. 7 using the experimental results described in Section 3 for $n$ - and $p$-type films. From Fig. 7 , it becomes clear that for resistors rotated by an angle $\theta \neq 0$ or $\pi / 2$, a pseudo-Hall effect takes place, with a gauge factor that can be read from the Mohr's circle.

\subsection{Doped n-type nc-Si}

For a semiconductor with a multi-valley conduction band structure, like silicon, the major contribution to the piezoresistance of the $n$-type material comes from the so-called "electron transfer effect" consisting in a strain-dependent uneven distribution of free

carriers between different valleys in the first Brillouin zone. ${ }^{15,30,31}$ The effect is proportional to the mobility anisotropy characteristic of the carriers located in one valley. As far as $n$-type doped $\mathrm{Si}$ is concerned, there are 6 equivalent (at rest) valleys in the conduction band, located along the (100) and equivalent directions in the Brillouin zone. Assuming Boltzmann statistics for the electrons, one can obtain the following result, ${ }^{30,31}$

$$
M_{11}=-\frac{2 \delta}{3} ; \quad M_{12}=\frac{\delta}{3} ; \quad M_{44}=0
$$

In Eq. (10) 


$$
\delta=\frac{1-\xi}{1+2 \xi} \frac{\Xi_{u}}{k T}
$$

where $\Xi_{u}$ is the deformation potential, $\xi=\mu_{\perp} / \mu_{\|}$, and $\mu_{\perp}$ and $\mu_{\|}$are the principal values of the mobility tensor for electrons in one valley. As a test, inserting in Eq. (11) the values of $\xi \approx 5$ and $\Xi_{u}=8.77 \mathbf{e V}$, given in Ref. 33 for c-Si, and substituting in Eq. (10) yields results that are in good agreement with the data of Table I.

Let us now consider the effect of this mechanism for a nc-Si film. We assume that the film consists of randomly oriented $n$-type Si crystallites that are sufficiently large to exclude any quantum size and surface scattering effects on the conductivity within each crystallite. Moreover, we assume that the influence of grain boundaries on the piezoresistance properties can be neglected because of the low inter-grain barrier height in multicrystalline silicon. ${ }^{26}$ The (random) orientation of a given crystalline grain with respect to the principal axes of the applied strain can be described by the three Euler angles $(\alpha, \beta, \gamma)$ as shown in Fig.2-b). Assuming that the local strain in the crystallite is equal to the average strain in the film, we can transform the macroscopic strain tensor, of the form

$$
\hat{\varepsilon}=\left(\begin{array}{ccc}
\varepsilon_{1} & 0 & 0 \\
0 & \varepsilon_{2} & 0 \\
0 & 0 & \varepsilon_{3}
\end{array}\right),
$$

to the local basis of the crystallite, $\left(x^{\prime}, y^{\prime}, z^{\prime}\right)$, according to $\hat{\varepsilon}^{\prime}=\vec{U}_{\alpha \beta \gamma} \hat{\varepsilon} \vec{U}_{\alpha \beta \gamma}^{-1}$, where $\vec{U}_{\alpha \beta \gamma}$ is a transformation matrix. ${ }^{32}$ Therefore we can find the strain-induced change in the crystallite conductivity,

$$
\Delta \hat{\sigma}^{\prime}=\hat{M}^{\prime} \hat{\varepsilon}^{\prime}=\frac{\delta}{3}\left(\begin{array}{ccc}
-2 \varepsilon_{x x}^{\prime}+\varepsilon_{y y}^{\prime}+\varepsilon_{z z}^{\prime} & 0 & 0 \\
0 & \varepsilon_{x x}^{\prime}-2 \varepsilon_{y y}^{\prime}+\varepsilon_{z z}^{\prime} & 0 \\
0 & 0 & \varepsilon_{x x}^{\prime}+\varepsilon_{y y}^{\prime}-2 \varepsilon_{z z}^{\prime}
\end{array}\right)
$$


and transform back to the laboratory frame. The last step consists in averaging the obtained (non-diagonal) $\Delta \sigma_{i j}$ tensor over the Euler angles,

$$
\left\langle\frac{\Delta \sigma_{i j}}{\sigma}\right\rangle=\frac{1}{\sigma} \frac{1}{8 \pi^{2}} \int_{0}^{\pi} \int_{0}^{2 \pi} \int_{0}^{2 \pi} \Delta \sigma_{i j}(\alpha, \beta, \gamma) \sin \beta d \beta d \alpha d \gamma
$$

This procedure provides a mean local conductivity which is prescribed to the whole film, within the spirit of a mean-field theory. The result is:

$$
\begin{aligned}
& \frac{\left\langle\Delta \sigma_{x x}\right\rangle}{\sigma}=\frac{2 \delta}{15}\left(\varepsilon_{2}+\varepsilon_{3}-2 \varepsilon_{1}\right) ; \\
& \frac{\left\langle\Delta \sigma_{y y}\right\rangle}{\sigma}=\frac{2 \delta}{15}\left(\varepsilon_{1}+\varepsilon_{3}-2 \varepsilon_{2}\right) ; \\
& \frac{\left\langle\Delta \sigma_{z z}\right\rangle}{\sigma}=\frac{2 \delta}{15}\left(\varepsilon_{1}+\varepsilon_{2}-2 \varepsilon_{3}\right),
\end{aligned}
$$

and all the non-diagonal components vanish. As expected, the principal axes of $\Delta \hat{\sigma}$ coincide with those of the strain tensor. The above result can be written in terms of an average piezoresistance tensor whose components, $\left\langle M_{11}\right\rangle$ and $\left\langle M_{12}\right\rangle$, are equal to $2 / 5$ of the corresponding values for c-Si.

Using the definitions (6) and Eqs. (14) - (16), we obtain for the gauge factors:

$$
\begin{array}{r}
G F_{L}=\frac{2 \delta}{15}\left(2-\frac{\varepsilon_{2}+\varepsilon_{3}}{\varepsilon_{1}}\right) \\
G F_{T}=-\frac{2 \delta}{15}\left(1-2 \frac{\varepsilon_{2}}{\varepsilon_{1}}+\frac{\varepsilon_{3}}{\varepsilon_{1}}\right) .
\end{array}
$$

For a thin film on a substrate submitted to pure bending we take:

$$
\varepsilon_{2}=-v \varepsilon_{1} ; \quad \varepsilon_{3}=0
$$


where $v$ is some effective value of the Poisson's ratio still obeying the general condition $v<1 / 2$. Using the values of $v \approx 0.25$ and $\xi, \Xi$ for c-Si from Ref. 33, we obtain, at room temperature, $G F_{L} \approx-39$ and $G F_{T} \approx 26$. While the first of these values is quite close to the experimental data, the transverse $G F$ is not predicted correctly by the model. This can be of course manifest of an excessive simplicity of the model. However, it can also be due to the fact that the strain in the vicinity of the resistors made along $\theta \neq 0$, under sample's bending can deviate significantly from the simple traction. Indeed, if we assume that for the micro-resistor with $\theta=\pi / 2, \varepsilon_{2} \approx \varepsilon_{1}, \varepsilon_{3}=0$ instead of (19), we obtain from (17) and (18) the result $G F_{L} \approx G F_{T}<0$.

\subsection{Doped p-type nc-Si}

The piezoresistance of $p$-type semiconductors with diamond or zinc-blend structure is due to the lifting of the degeneracy of the light and heavy-hole sub-bands in the center of the Brillouin zone. Change in the conductivity related to each type of holes comes from several deformation-caused effects, such as changes in the distribution function of the particles over energies, as well as in their group velocities and relaxation times. ${ }^{30}$ Even though it is possible to derive a microscopic expression for $\hat{M},{ }^{30}$ it is rather complex and contains several parameters that are not well known. Therefore we shall simply use the empirical values of c-Si (see Table I) as input parameters for our model. Thus, we assume:

$$
M_{11}=M_{12}=0 ; \quad M_{44}=-\pi_{44} C_{44} \equiv \mathrm{M} .
$$

Instead of Eq. (12), we have now

$$
\Delta \hat{\sigma}^{\prime}=\mathrm{M}\left(\begin{array}{ccc}
0 & \varepsilon_{x y}^{\prime} & \varepsilon_{x z}^{\prime} \\
\varepsilon_{x y}^{\prime} & 0 & \varepsilon_{y z}^{\prime} \\
\varepsilon_{x z}^{\prime} & \varepsilon_{y z}^{\prime} & 0
\end{array}\right),
$$


which means that the conductivity change in each crystallite is produced only by shear strains (for example, grains with the crystal axes parallel to the laboratory frame do not contribute to the piezoresistance). Using the same procedure as in 4.2, we obtain:

$$
\begin{aligned}
& \frac{\left\langle\Delta \sigma_{x x}\right\rangle}{\sigma}=-\frac{\mathrm{M}}{5}\left(\varepsilon_{2}+\varepsilon_{3}-2 \varepsilon_{1}\right) ; \\
& \frac{\left\langle\Delta \sigma_{y y}\right\rangle}{\sigma}=-\frac{\mathrm{M}}{5}\left(\varepsilon_{1}+\varepsilon_{3}-2 \varepsilon_{2}\right) ; \\
& \frac{\left\langle\Delta \sigma_{z z}\right\rangle}{\sigma}=-\frac{\mathrm{M}}{5}\left(\varepsilon_{1}+\varepsilon_{2}-2 \varepsilon_{3}\right) .
\end{aligned}
$$

This is essentially the same result as for $n-\mathrm{nc}-\mathrm{Si}$, with the replacement $(-2 \delta / 3) \rightarrow \mathrm{M}$. Taking $C_{44}=0.8 \times 10^{11} \mathrm{~Pa}$, we have $\mathrm{M} \approx-110$ and for the gauge factors under the same strain field (19) we obtain $G F_{L} \approx 49.5$ and $G F_{T} \approx-31$. The model slightly overestimates the longitudinal effect and, like in the case of $n$-type material, the sign of the transverse $G F$ is not predicted correctly, although negative $G F_{T}$ values for hydrogenated microcrystalline silicon have been reported in the literature. ${ }^{16}$

\section{Conclusions}

The piezoresistive response of $n$ - and $p$-type nanocrystalline silicon thin films deposited by HW-CVD, RF-PECVD and VHF-PECVD was measured in lithographically defined micro-resistors using four-point bending tests. Negative gauge factors were found, both longitudinal and transverse, for $n$-type samples while $p$-type films show positive $G F$ s. All films have isotropic piezoresistive response in planes parallel to the surface and the principal axes of the piezoconductivity tensor coincide with those of the strain tensor. The isotropy of the piezoresistive response in directions parallel to the film free surface was confirmed by measurements of the $G F \mathrm{~s}$ on rotated resistors for different angles with 
respect to the direction of the applied stress. The $G F$ values have a sinusoidal dependence on the angle $\theta$, similar to the angular dependence of the normal strain as can be illustrated by a Mohr circle. A deviation from such behavior would be manifest of structural anisotropy of the film. However, in none of the films studied in this work was this deviation significant.

A mean-field model for the piezoresistivity of nc-Si films assuming random orientation of crystalline grains and neglecting effects of grain boundaries was developed. The model predicts that the longitudinal piezoresistance of nanocrystalline films can reach $40 \%$ of its maximum value for c-Si. While in the crystalline material the effect is strongly anisotropic (for instance, it nearly vanishes for $p$-type c-Si under traction along one of its crystal axes, $\left.\pi_{11} \approx 0\right)$, the effect is isotropic in nanocrystalline films making them a suitable material for sensors of tensile strains applied in arbitrary direction. This theoretical conclusion agrees very well with the experimental data, even though the model fails in reproducing the measured values of the transverse gauge factor. One possible reason for this can be the importance of grain boundary effects, particularly in the direction perpendicular to the applied tensile strain. Another possibility is that the strain field in the vicinity of the micro-resistors with $\theta \neq 0$ can deviate substantially from the assumed simple form (19). Further experimental and theoretical efforts are required in order to clarify these issues.

\section{Acknowledgments}

This work was partially supported by the Portuguese Foundation for Science and Technology (FCT) under grant PTDC/CTM/66558/2006. One of the authors (PA) acknowledges FCT (research grant SFRH/BSAB/883/2009) and IMTEK-Freiburg for 
support during his sabbatical leave. The authors acknowledge B. Winter from ipe for thermal oxidation of 4 " wafers. 


\section{References}

${ }^{1}$ P. Alpuim, V. Chu and J. P. Conde, J. Appl. Phys. 86, 3812 (1999).

${ }^{2}$ G. N. Parsons, C.-S. Yang, T. M. Klein, and L. Smith, Mat. Res. Soc. Symp. Proc. 507, 19 (1998).

${ }^{3}$ V. Terrazzoni-Daudrix, F.-J. Haug, T. Söderström, C. Ballif, D. Fischer, W. Soppe, J. Bertomeu, M. Fahland, H.Schlemm, M. Grimm, M. Topic and M. Wutz, Proceedings of the 24th European Photovoltaic Solar Energy Conference, 21-25 September 2009, Hamburg, Germany.

${ }^{4}$ M. B. Schubert, Thin Solid Films 337, 240 (1999).

${ }^{5}$ G. Parascandolo, G. Bugnon, A. Feltrin and C. Ballif, Progress in Photovoltaics: Research and Applications 18, 257 (2010).

${ }^{6}$ S. Bae and S. J. Fonash, J. Vac. Sci. Technol. A 17, 1987 (1999).

${ }^{7}$ P. Roca i Cabarrocas, Y. Djeridane, Th. Nguyen-Tran, E. V. Johnson, A. Abramov and Q Zhang, Plasma Phys. Control. Fusion 50, 124037 (2008).

${ }^{8}$ J. Meier, R. Fluckiger, H. Keppner and A. Shah, Appl. Phys. Lett. 65, 860 (1994).

${ }^{9}$ N. Kosku and S. Miyazaki, Thin Solid Films 511-512, 265 (2006).

${ }^{10}$ Haijun Jia, J. K. Saha and H. Shirai, Jpn. J. Appl. Phys. 45, 666 (2006).

${ }^{11}$ H. Matsumura, Y. Tashiro, K. Sasaki and S. Furukawa, Jpn. J. Appl. Phys. 33, L1209 (1994).

${ }^{12}$ S. A. Filonovich, P. Alpuim, L. Rebouta, J.-E. Bourée and Y. M. Soro, Journal of NonCrystalline Solids 354, 2376 (2008).

${ }^{13}$ W. G. Pfann and R. N. Thurston, J. Appl. Phys. 32, 2008 (1961).

${ }^{14}$ O. N. Tufte, P.W. Chapman and D. Long, J. Appl. Phys. 33, 3322 (1962).

${ }^{15}$ C. S. Smith, Phys. Rev. 94, 62 (1954).

${ }^{16}$ S. Nishida, M. Konagai and K. Takahashi, Jpn. J. Appl. Phys. 25, 17 (1986).

${ }^{17}$ W. Germer, Sensors and Actuators 7, 135 (1985).

${ }^{18}$ M. Utsunomiya and A. Yoshida, Applied Surface Science 33-34, 1222 (1988).

${ }^{19}$ P. Alpuim, V. Chu and J.P. Conde, IEEE Sens. J. 2, 336 (2002).

${ }^{20}$ V. Lumelsky, M. Shur, and Sigurd Wagner, IEEE Sensors Journal 1, 41 (2001).

${ }^{21}$ See, for example, M. Elwenspoek and R. Wiegerink, Mechanical Microsensors (Springer, New York, 2001), p. 87 or S. Middelhoek and S. A. Audet, Silicon Sensors" (Delft, The Netherlands: Delft Univ. Press, 1994), pp. 95-193.

${ }^{22}$ O. Paul, J. Gaspar, and P. Ruther, IEEE Trans. Elect. Electron. Eng. 2, 199 (2007). 
${ }^{23}$ J. Gaspar, V. Chu, and J. P. Conde, IEEE J. Microelectromech. Syst. 14, 1082 (2005).

${ }^{24}$ J. Gaspar, H. Li, P. P. Freitas, V. Chu, and J. P. Conde, IEEE J. Microelectromech. Syst. 12, 550 (2003).

${ }^{25} \mathrm{H}$. Li, J. Gaspar, P. P. Freitas, V. Chu, and J. P. Conde, IEEE Trans. Magnetics 38, 3371 (2002).

${ }^{26}$ M. Eickhoff and M. Stutzmann, J. Appl. Phys. 96, 2878 (2004).

${ }^{27}$ F. I. Baratta, Commun. Am. Cer. Soc. C-86, May (1981).

${ }^{28}$ E. Lund and T. G. Finstada, Review of Scientific Instruments 75, 4960 (2004).

${ }^{29}$ See, for example, S. P. Timoshenko and J. N. Goodier, Theory of elasticity (McGrawHill, Singapore, 1970), pp. 19-22.

${ }^{30}$ G. L. Bir and G. E. Pikus, Symmetry and Strain-Induced Effects in Semiconductors (Wiley, New York, 1974), Chapter VI.

${ }^{31}$ C. Herring and E. Vogt, Phys. Rev. 101, 944 (1956).

${ }^{32}$ See, for example, G. A. Korn and T. M. Korn, Mathematical Handbook for Scientists and Engineers (McGraw-Hill, New York, 1968), p. 450.

${ }^{33}$ A. Blacha, H. Presting, M. Cardona, "Deformation Potentials of $k=0$ States of Tetrahedral Semiconductors”, Phys. Stat. Sol. (b) 126 (1984) 11. 
Table I. Piezoresistance coefficients of crystalline silicon (adapted from Ref. 15)

\begin{tabular}{|l|l|l|l|}
\hline & $\pi_{11}\left(\times 10^{-11} \mathrm{~Pa}^{-1}\right)$ & $\pi_{12}\left(\times 10^{-11} \mathrm{~Pa}^{-1}\right)$ & $\pi_{44}\left(\times 10^{-11} \mathrm{~Pa}^{-1}\right)$ \\
\hline$n$-type Si & -102 & 53 & -14 \\
\hline$p$-type Si & 7 & -1 & 138 \\
\hline
\end{tabular}

Table II. Summary of deposition conditions and properties of studied films

\begin{tabular}{|c|c|c|c|c|c|c|c|c|c|c|}
\hline sample & $\begin{array}{c}\text { CVD / } \\
\text { wafer } \\
\text { size (") }\end{array}$ & $\begin{array}{c}T_{\text {sub }} \\
\left({ }^{\circ} \mathrm{C}\right)\end{array}$ & $\begin{array}{c}p_{\mathrm{w}} \\
(\text { Torr })\end{array}$ & $\begin{array}{c}H D \\
(\%)\end{array}$ & $\begin{array}{c}\text { Doping } \\
\text { type }\end{array}$ & $\begin{array}{c}d \\
(\mathrm{~nm})\end{array}$ & $\begin{array}{c}R_{0} \\
(\Omega)\end{array}$ & $\begin{array}{c}\sigma_{\mathrm{dk}} \\
\left(\Omega^{-1} \mathrm{~cm}^{-1}\right)\end{array}$ & $G_{L}$ & $G F_{T}$ \\
\hline$n$-RF\#01 & RF 4" & 250 & 0.800 & 99 & $\mathrm{n}$ & 200 & 589.7 & 25.4 & -25.2 & -8.40 \\
\hline$p$-RF\#02 & RF 4" & 280 & 0.650 & 77 & $\mathrm{p}$ & 170 & $1.98 \times 10^{8}$ & $5.6 \times 10^{-5}$ & 24.4 & 7.08 \\
\hline$n$-RF\#03 & RF 2" & 220 & 1.5 & 98.5 & $\mathrm{n}$ & 160 & 1605 & 8.5 & -23.9 & -5.40 \\
\hline$n$-RF\#04 & RF 2" & 150 & 3 & 98.5 & $\mathrm{n}$ & 200 & 1488 & 9.7 & -28.0 & -5.60 \\
\hline$p$-VHF\#05 & VHF 4” & 250 & 0.250 & 98 & $\mathrm{p}$ & 200 & 9569 & 1.63 & 41.0 & 2.84 \\
\hline$p$-VHF\#06 & VHF 4" & 250 & 0.250 & 97 & $\mathrm{p}$ & 200 & 25011 & 0.60 & 34.6 & 3.80 \\
\hline$n$-HW\#07 & HW 2" & 150 & 0.040 & 90 & $\mathrm{n}$ & 150 & 3329 & 2.87 & -17.4 & -6.24 \\
\hline$p$-HW\#08 & HW 2" & 150 & 0.040 & 90 & $\mathrm{p}$ & 315 & $4.50 \times 10^{4}$ & 0.212 & 25.0 & 5.2 \\
\hline$p$-HW\#09 & HW 2" & 150 & 0.040 & 95 & $\mathrm{p}$ & 300 & 3500 & 2.8 & 32.1 & 7.2 \\
\hline
\end{tabular}




\section{Figure Captions}

Fig. 1 - a) Close-up of device under test (DUT) showing six resistors rotated by different angles relative to the longitudinal axis of the Si strip. b) DUT wire bonded to a printed circuit board. c) Four-point bending bridge with silicon strip mounted setup.

Fig. $2-$ a) Schematics of the four-point bending set up. $L_{o}$ is the distance between the first and second loading bars. $L_{o}+L_{i}$ is one half the distance between the outward loading bars. b) Simplified view of the load configuration and the multicrystalline system studied, showing a randomly oriented crystallite and defining the Euler angles.

Fig. 3 Relative resistance change as a function of applied strain for: a) $p$-type and, b) $n$ type nc-Si:H films deposited by HW ( $p$-HW\#09) and RF ( $n$-RF\#04), respectively, at $T_{\text {sub }}=$ $150^{\circ} \mathrm{C}$.

Fig. 4 - Column chart with error bars of the longitudinal (open columns) and transverse (striped columns) gauge factors for $n$ - and $p$-type nc-Si:H films studied in this work. $n$-type films have $G F<0$ and $p$-type films have $G F>0$.

Fig. 5 - a) Relative resistance change in $n$-type nc-Si:H piezoresistors as a function of principal strain, $\varepsilon_{1}$, for resistors aligned at angles $\theta=0^{\circ}, 30^{\circ}, 45^{\circ}, 90^{\circ}$ with the principal strain axis (lines are guides to the eye). $R_{0}$ is the resistance of the undeformed resistor. $\mathrm{b}$ ) $G F$ plotted as a function of $\theta$ (solid marks) and fitting with a constant plus a cosine function (line). The fitting parameters are given in the inset. The nc-Si:H thin film was deposited by RF-PECVD at $T_{\text {sub }}=250^{\circ} \mathrm{C}$. 
Fig. 6 - a) Relative resistance change in $p$-type nc-Si:H piezoresistors as a function of principal strain, $\varepsilon_{1}$, for resistors aligned at angles $\theta=0^{\circ}, 30^{\circ}, 45^{\circ}, 90^{\circ}$ with the principal strain axis (lines are guides to the eye). $R_{0}$ is the resistance of the undeformed resistor. $\mathrm{b}$ ) $G F$ plotted as a function of $\theta$ (solid marks) and fitting with a constant plus a cosine function (line). The fitting parameters are given in the inset. The nc-Si:H thin film was deposited by VHF-PECVD at $T_{\text {sub }}=250^{\circ} \mathrm{C}$.

Fig. 7 - Mohr's circles for GFs in $n^{+}$-nc-Si:H films, $n$-RF\#01 (solid line) and $n$-HW\#07 (dotted line), and in $p^{+}$-nc-Si:H film, $p$-VHF\#05 (dashed line). The radius and the center of the circles were calculated in a similar way to the one used when drawing a Mohr circle for strain. 


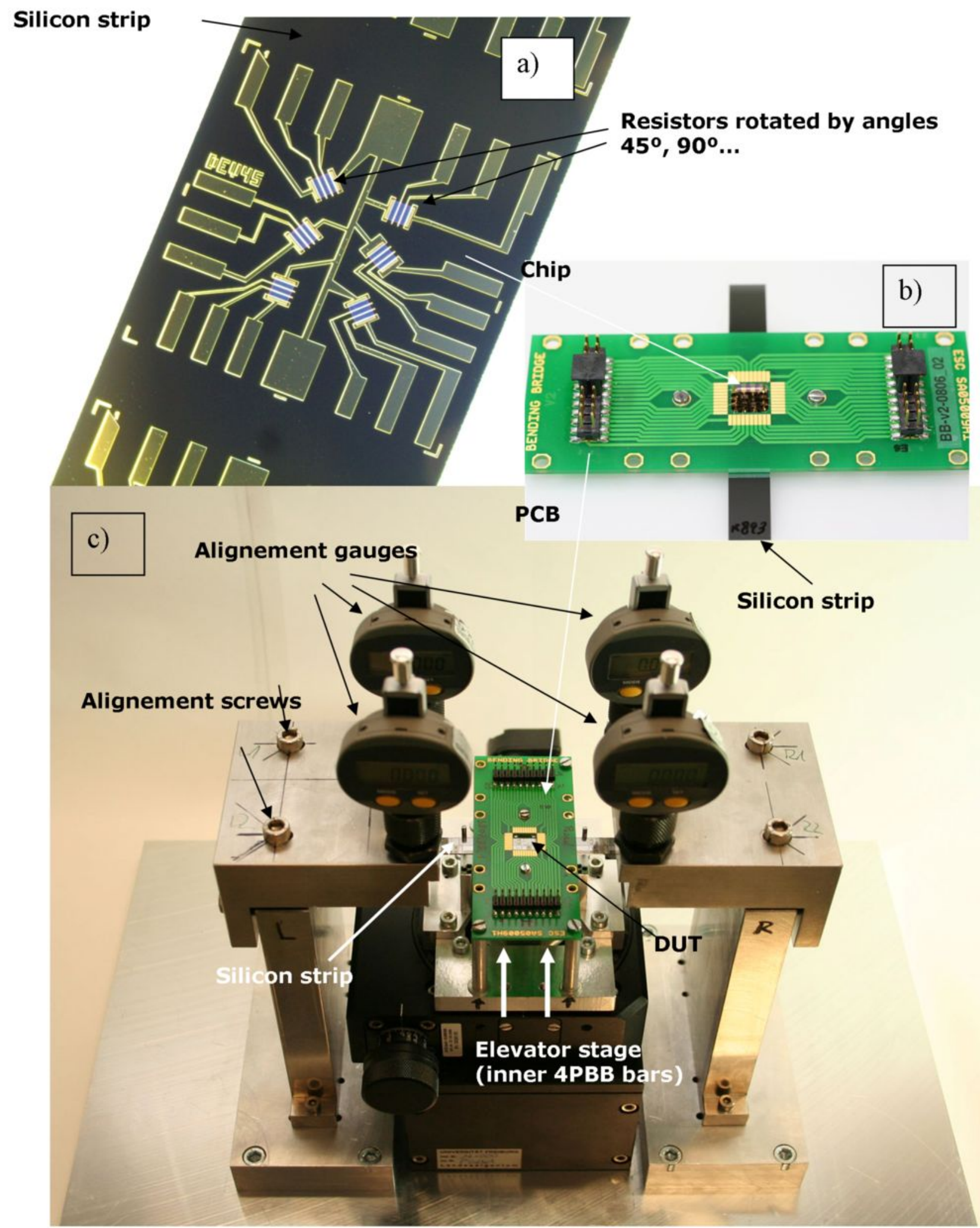


Figure 2

a)

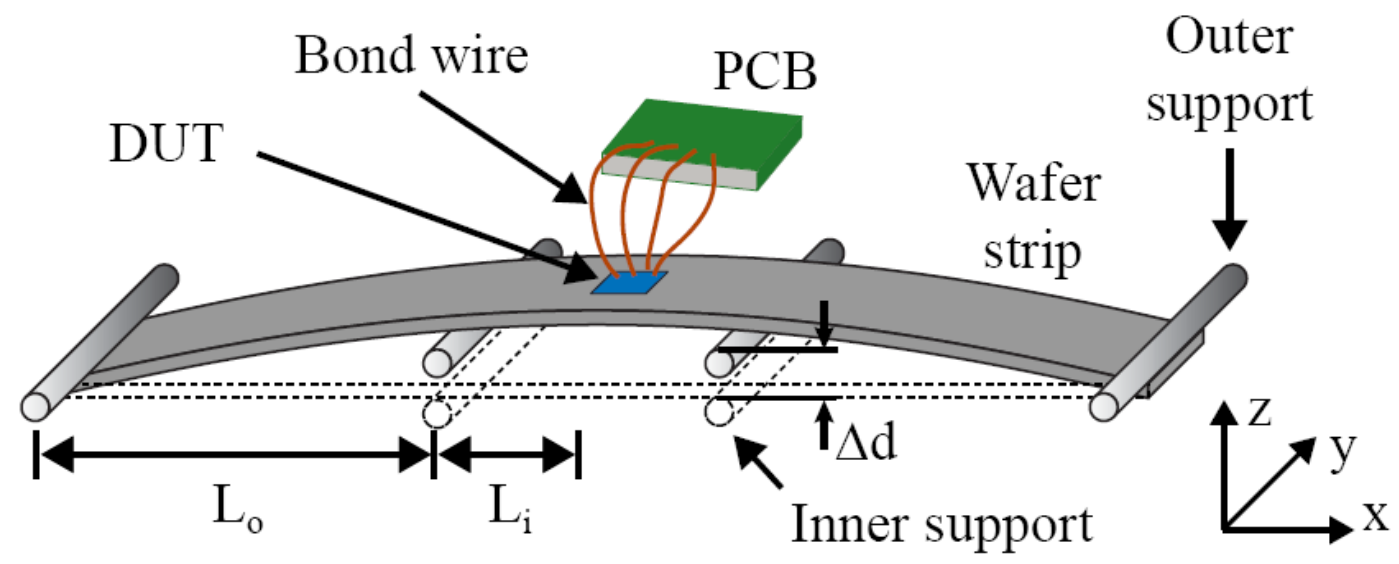

b)

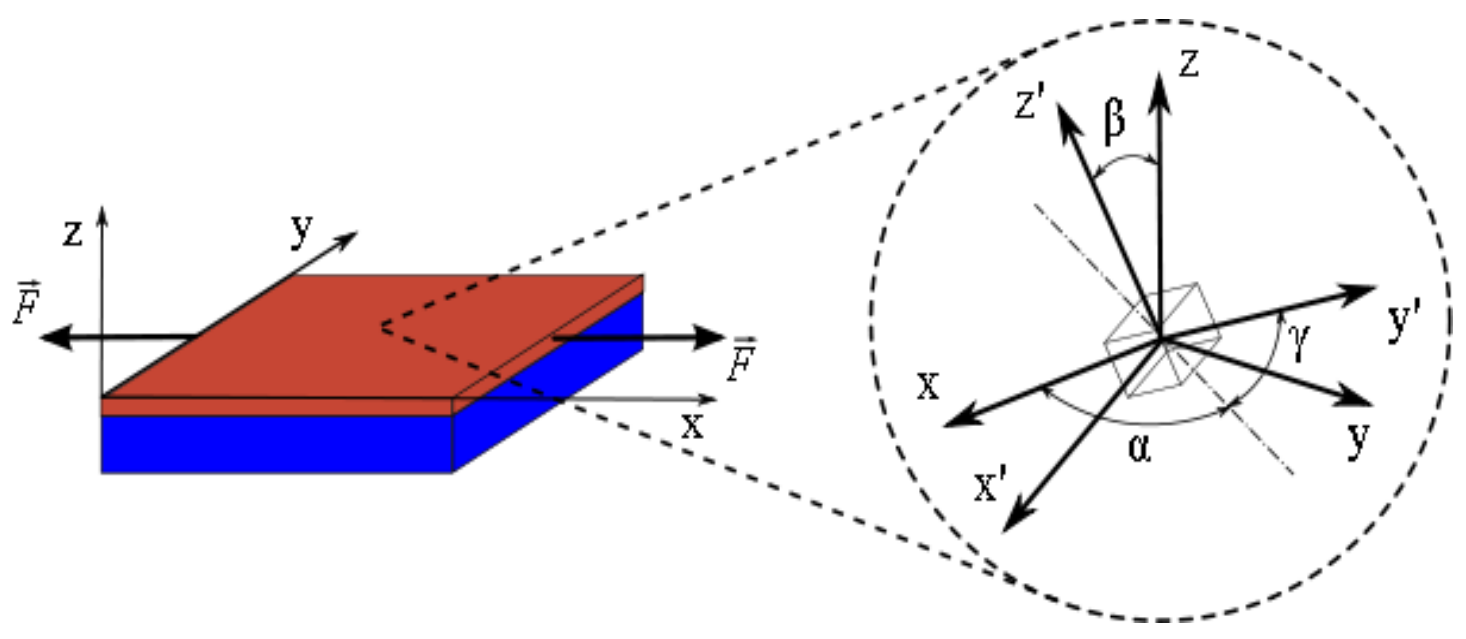




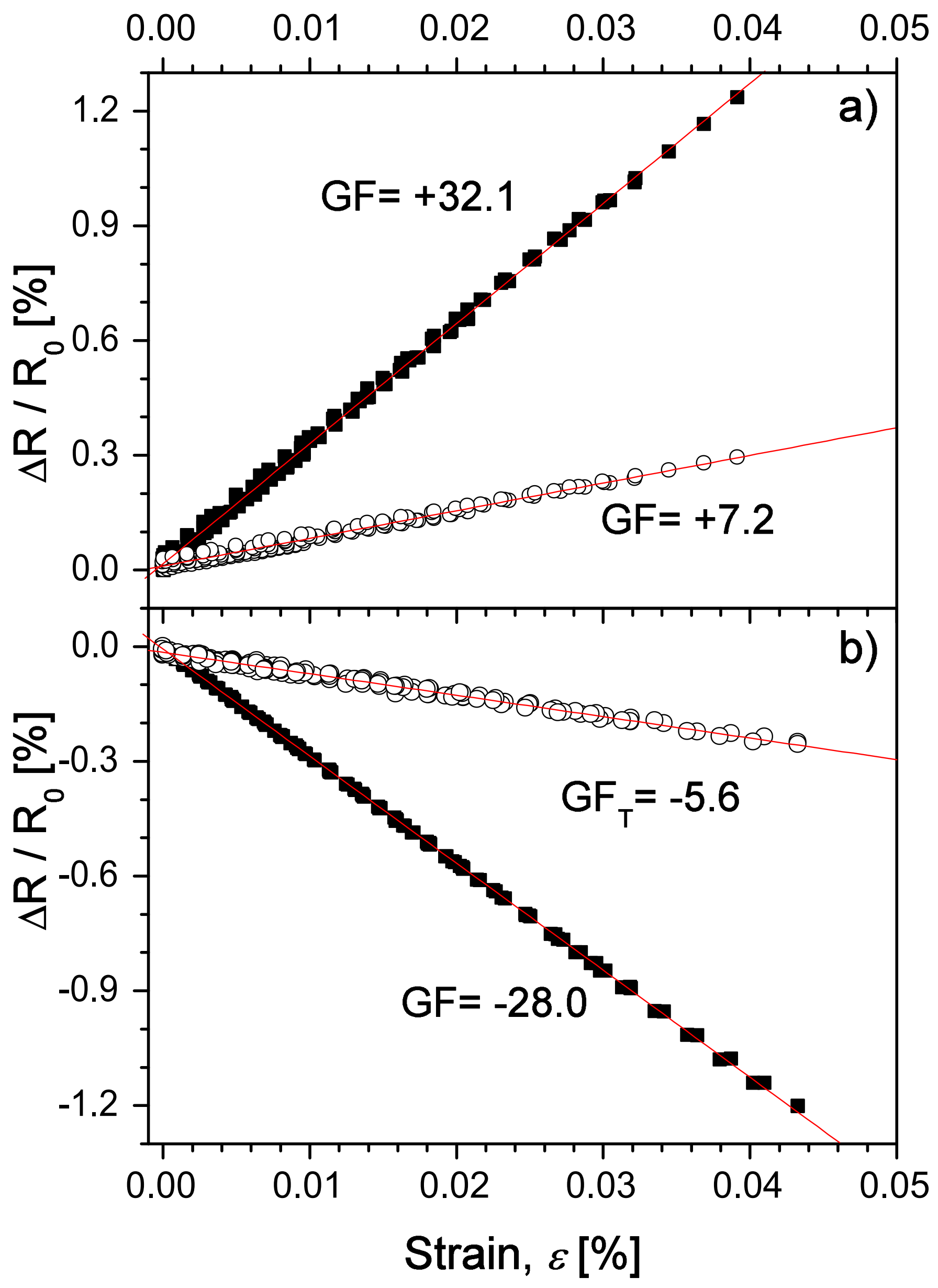




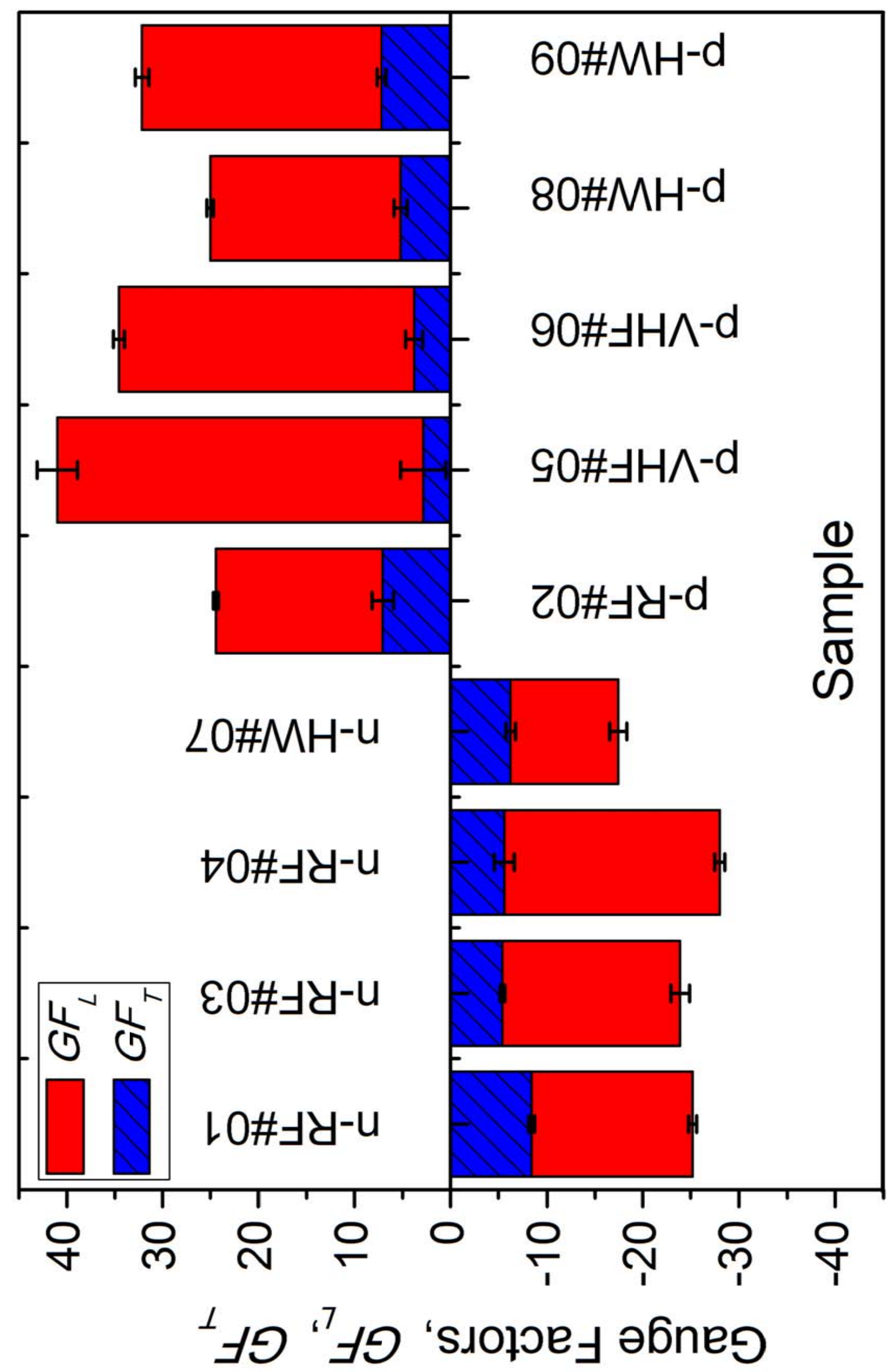



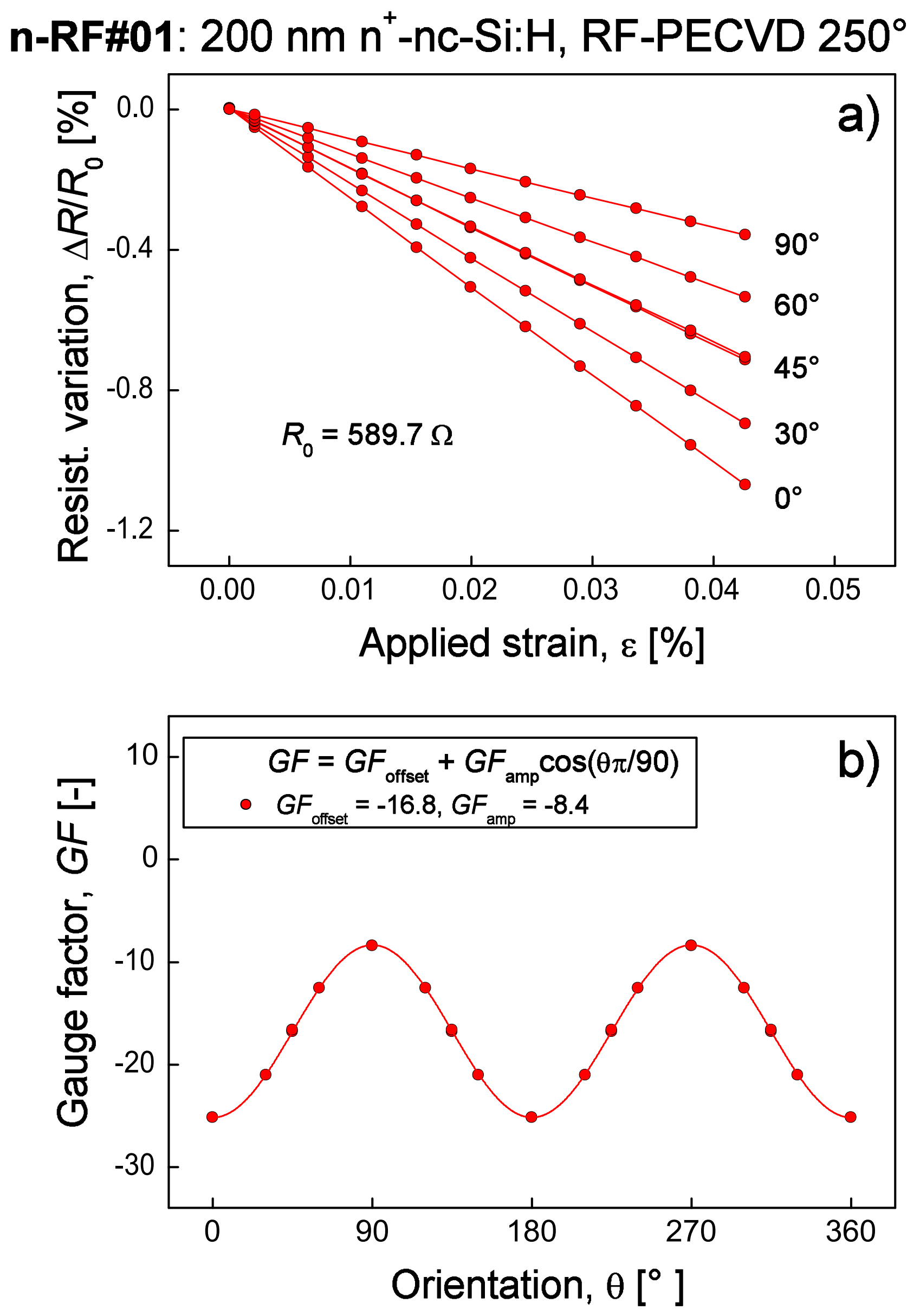
p-VHF\#05: 200 nm p ${ }^{+}$-nc-Si:H, PECVD 250 C
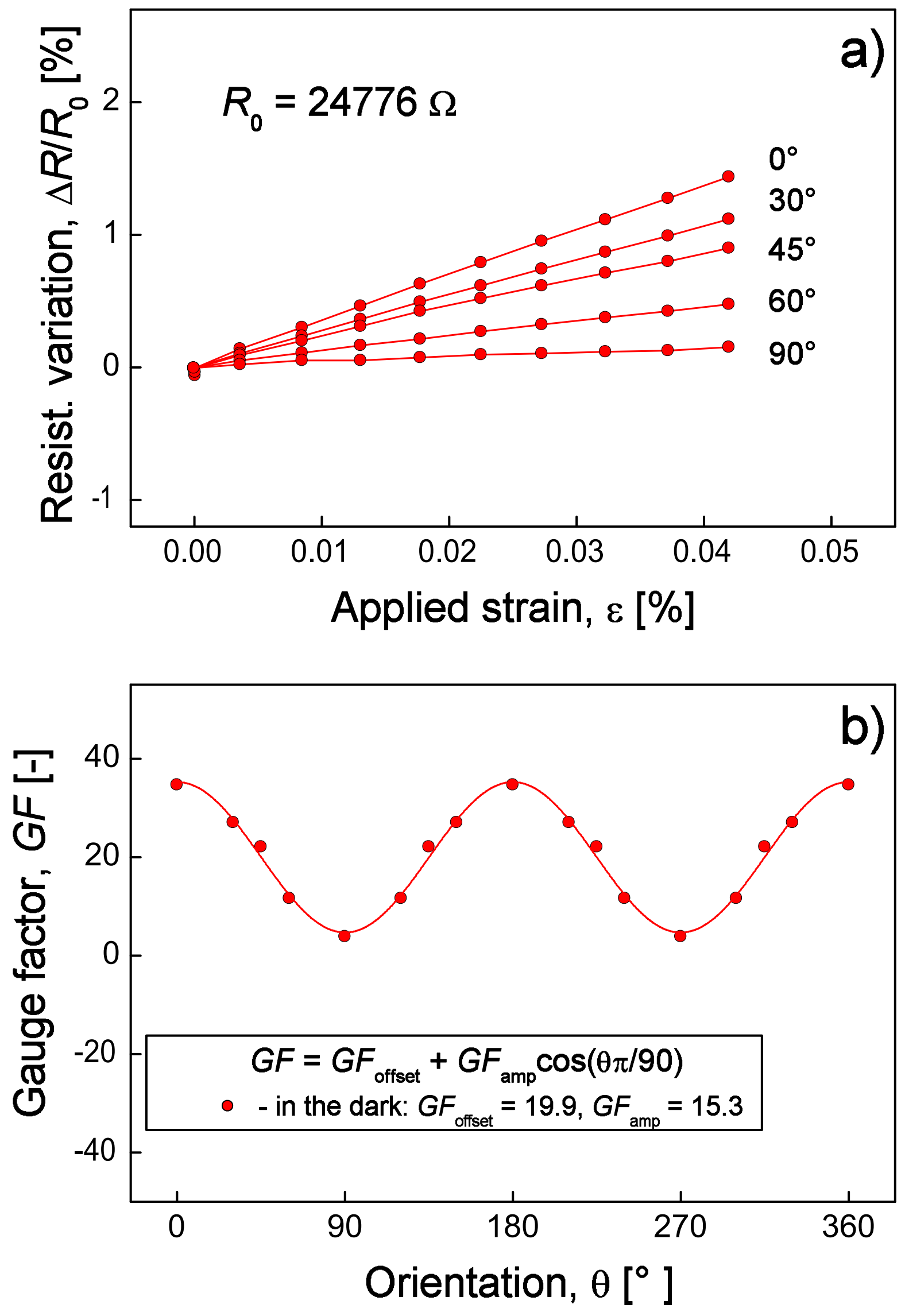


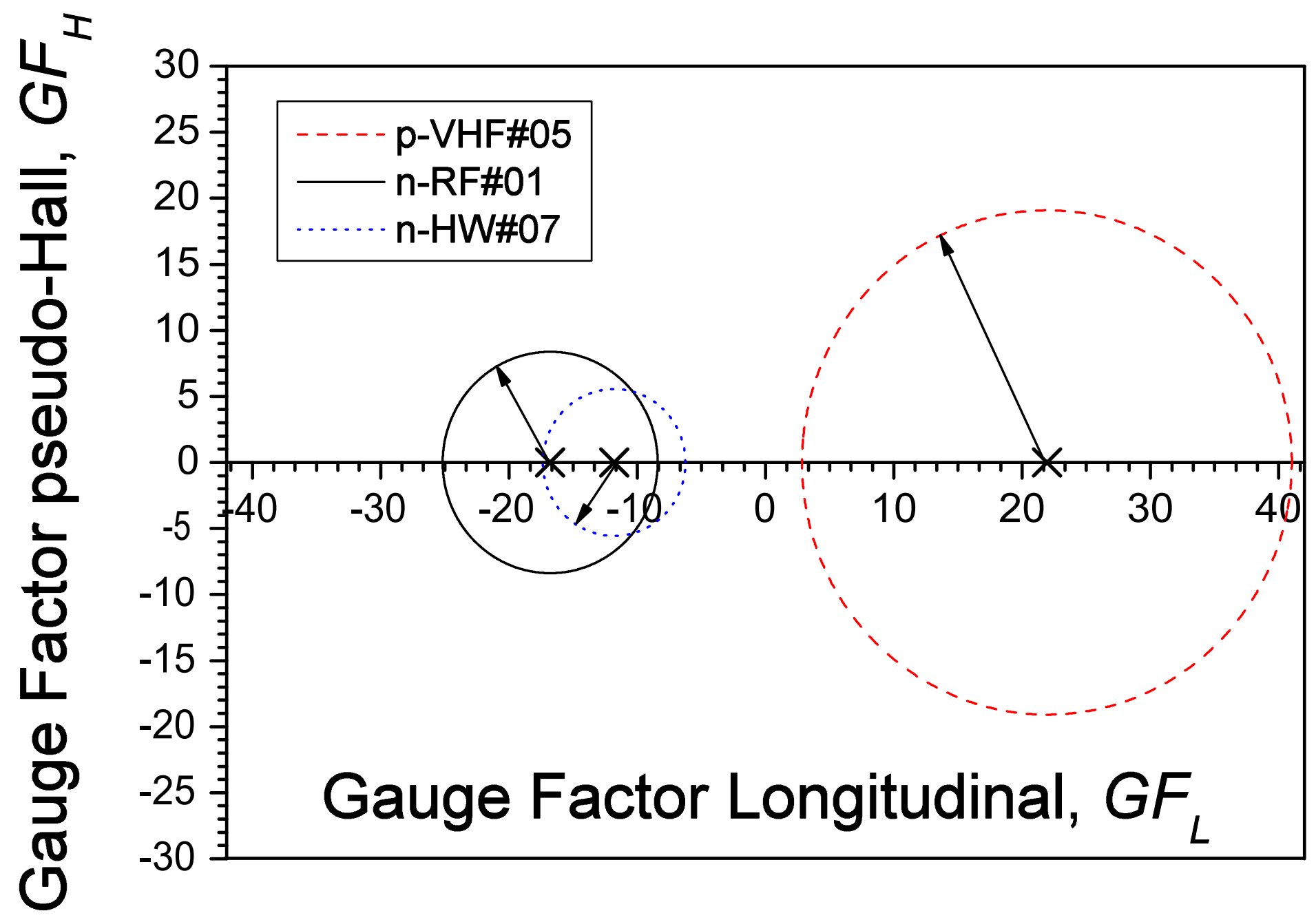

